\title{
ROTATING STELLAR INTERIORS \\ (Review Paper)
}

Robert Connon Smith

Astronomy Centre, University of Sussex, Falmer, Brighton BN1 9QH, U.K.

\begin{abstract}
Rotation has two main qualitative effects on the structure of a star: it distorts the shape and it reduces the total luminosity. The luminosity depends only on the total angular momentum but the shape, and so the observable properties, depends on the internal distribution of angular momentum. The expected distribution, even for main-sequence stars, is an unsolved problem. Redistribution of angular momentum can occur as a result of large-scale circulation currents, instabilities and magnetic stresses and there may be no steady state. If shear instabilities are dominant, then stars may rotate nearly uniformly. Studies of non-radial oscillations might perhaps eventually lead to a direct probing of the internal angular momentum distribution by the methods now being used to study the internal rotation of the Sun. Differential rotation near the surface would probably give rise to turbulent motions, which could generate the mechanical energy flux which seems to be a necessary input to the winds in hot stars.
\end{abstract}

\section{INTRODUCTION}

Interest in rotating stars began with Galileo's discovery of the Sun's rotation, revealed by the motion of sunspots (1612), but it was some three hundred years before Schlesinger (1910) showed that other stars also rotate. The observer measures the rotation speed of a star by the broadening of the spectral lines, usually interpreting the Doppler half-width in terms of a projected equatorial rotation speed $v \sin i$, where $i$ is the angle of inclination between the rotation axis and the line of sight. For real stars, the quantity $v$ is only approximately related to the linear rotation speed at the equator, $v_{\mathrm{e}}$ (Collins 1986).

Theoretically, the main effect of rotation is to distort a star from being spherically symmetric to being axially symmetric and a more useful theoretical measure of the rotation speed is $\alpha$, the ratio of the centrifugal force to gravity, measured at 
the surface equator. For a star of mass $M$, equatorial angular velocity $\Omega_{\mathrm{e}}$ and equatorial radius $R_{\mathrm{e}}, \alpha=\Omega_{\mathrm{e}}^{2} R_{\mathrm{e}}^{3} / G M$. This quantity is a measure of the distortion from spherical symmetry and is observable, at least in principle. In terms of $\alpha$, most stars are very slowly rotating; the Sun has $\alpha \simeq 2,10^{-5}$ and even the Crab pulsar, with a rotation period of $30 \mathrm{~ms}$, has $\alpha \simeq 3 \times 10^{-3}$. For this reason, the classical theory of stellar structure and evolution, in which stars are taken to be spherically symmetrical, has been very successful in describing the behaviour of the majority of stars.

Be stars are exceptional in having $\alpha \sim \mathrm{O}(1)$. Although this does not mean that they will spontaneously throw off a ring of material, as was once imagined, it does mean that rotational effects are not just a perturbation but may have a significant effect on the internal structure and dynamics of Be stars. We must therefore understand the general effects of rotation on stellar structure if we are fully to understand Be stars.

The theory of stellar rotation began as a mathematical problem that attracted many able mathematicians (Maclaurin, Jacobi, Darwin, etc.). The classical work on rotating liquids has been surveyed and extended by Chandrasekhar (1969); although real stars are not liquids, this work is still of relevance to problems in cosmogony, in particular the fission theory of binary stars (cf. Lebovitz 1975), and has been reviewed by Lebovitz $(1967,1979)$ and by Durisen \& Tohline (1985). I shall not discuss it here.

The most useful single reference work on rotating stellar interiors is the book by Tassoul (1978), which gives a remarkably complete survey of the field up to 1976 , with a few later references. Other useful reviews include Mestel (1965) on meridional circulation, Strittmatter (1969) on general theoretical developments, Fricke \& Kippenhahn (1972) on the evolution of rotating stars (see also my review in the present volume), Acheson (1978) on instabilities and Moss \& Smith (1981) on most aspects of stellar rotation and magnetic stars. In this article I shall concentrate on the effects of rotation on the internal structure of a star, relating the results wherever possible to the specific problem of the Be stars. I shall therefore consider only stars with a convective core and a radiative envelope (i.e. with $\left.M>1.5 M_{\odot}\right)$.

\section{THE MAXIMUM VALUE OF $\alpha$}

Although $\mathrm{Be}$ stars rotate rapidly, none is observed to have $\alpha=1$ and in fact the maximum observed value of $\alpha$ is about 0.8 (cf. Slettebak 1976 and this volume). Does this mean that no Be star rotates at its critical velocity? Theoretically, it is obvious that $\alpha \leq 1$ and I expect that most people believe that $\alpha_{\max }=1$. However, it turns out that that is only true if $\Omega$ is a constant or 
increases from pole to equator $\left(\Omega_{\mathrm{p}}<\Omega_{\mathrm{e}}\right)$. If $\Omega_{\mathrm{p}}>\Omega_{\mathrm{e}}$, then $\alpha_{\max }<1$. For example (see Appendix), $\alpha_{\max } \simeq 0.7$ for the case $\Omega=\Omega_{\mathrm{p}}(1-0.2 x)^{2}$, which corresponds to $\Omega_{\mathrm{p}} \simeq 1.5 \Omega_{\mathrm{e}}$. Thus if we had a star for which we deduced using $\alpha=1$ that " $v_{\text {crit }} "=550 \mathrm{~km} \mathrm{~s}^{-1}$ then the actual maximum observable rotation speed would be $v_{\max } \simeq(0.7)^{1 / 2} 550 \mathrm{~km} \mathrm{~s}^{-1} \simeq 460 \mathrm{~km} \mathrm{~s}^{-1}:$ a large effect. Although this result depends on using the Roche approximation for the gravitational potential, I expect it to be qualitatively correct.

What are the consequences of this? I believe that there are two deductions that we can make from the observed rotation speeds.

First, the fact that $(v \sin i)_{\max }($ observed $)<v_{\text {crit }}(\alpha=1)$ now has two possible interpretations: either Be stars are not rotating at their maximum possible speeds (the usual interpretation) or Be stars do not rotate uniformly (and have $\Omega_{\mathrm{p}}>\Omega_{\mathrm{e}}$ ). Of course, both may be true, and we certainly cannot distinguish the possibilities from Doppler half-widths alone.

Second, it is clear that

$$
\alpha_{\max }=\left(\frac{v_{\max }}{v_{\text {crit }}}\right)^{2} \geq \frac{(v \sin i)_{\max }^{2}(\text { observed })}{v_{\text {crit }}^{2}}
$$

and so the maximum observed $v \sin i$ puts some constraints on the degree of differential rotation. I have not attempted to apply this constraint in detail, but the fact that at least some stars seem to have $\alpha>0.7$ (Slettebak 1976) suggests from my example that $\Omega_{\mathrm{p}}<1.5 \Omega_{\mathrm{e}}$.

\section{THE STRUCTURE EQUATIONS FOR ROTATING STARS}

Because the effect of rotation is to transform a star from being spherically-symmetric to being symmetric about the rotation axis, it is mathematically convenient to use a cylindrical polar coordinate system $(\tilde{\omega}, \phi, z)$. The equations of stellar structure become vector rather than scalar and we must include the time derivatives because, as we shall see, it is not clear that there is a unique steady solution of the kind that exists for spherical stellar models.

We first write down the $(\tilde{\omega}, z)$ components of the equation of motion, neglecting viscous and magnetic forces:

$$
\frac{\partial \mathbf{v}}{\partial t}+(\mathbf{v} \cdot \boldsymbol{\nabla}) \mathbf{v}+\frac{1}{\rho} \boldsymbol{\nabla} P=\nabla \Phi+\Omega^{2} \tilde{\boldsymbol{\omega}}
$$

where $\mathbf{V}$ is the total velocity of stellar material and we have written

$$
\mathbf{V}=\mathbf{v}+\Omega \tilde{\omega} \mathbf{t}
$$


where $\mathbf{t}$ is a unit vector in the $\phi$ direction and $\Omega$ is the angular velocity at distance $\tilde{\omega}$ from the rotation axis. $v$ is then the velocity in meridian planes, i.e. in planes through the rotation axis, and is often referred to as the meridian or meridional velocity. $P, \rho$ and $\Phi$ are pressure, density and gravitational potential respectively and are functions of $\tilde{\omega}$ and $z$. Because of axial symmetry, none of the terms in equation (1) has a $\phi$ component and so the $\phi$ component of the equation of motion consists simply of an unbalanced Coriolis force, which can be written as

$$
(\mathrm{v} . \nabla) \Omega \tilde{\omega}^{2}=0
$$

We shall return to the interpretation of this equation later.

If there are no internal motions other than rotation, and we assume a steady state, then equation (1) simplifies to

$$
\frac{1}{\rho} \nabla P=\nabla \phi+\Omega^{2} \tilde{\omega} .
$$

This is the generalisation of the usual hydrostatic equation $(1 / \rho)(d P / d r)=-G M / r^{2}$ for spherical stars. The gravitational potential is now given by Poisson's equation:

$$
\nabla^{2} \Phi=-4 \pi G \rho
$$

which is much more complicated than the spherical equivalent, $d M / d r=4 \pi r^{2} \rho$, and is particularly awkward to solve for highly-distorted stars.

If (and only if) $\Omega$ is a function only of $\tilde{\omega}$, the centrifugal force can be expressed as the gradient of a potential and (4) simplifies still further to

$$
\nabla P=\rho \nabla \Psi
$$

where $\Psi=\Phi+W$ and

$$
W=\int_{0}^{\tilde{\omega}} \Omega^{2} \tilde{\omega} d \tilde{\omega} .
$$

For uniform rotation, $W=\frac{1}{2} \Omega^{2} \tilde{\omega}^{2}$. Equation (6) implies that $P$ (and so $\rho$ ) is a function only of $\Psi$ and so surfaces of constant $\Psi$ play the same role in rotating stars as spheres do in non-rotating ones - they are the natural coordinate surfaces. Because these surfaces only exist when $\Omega=\Omega(\bar{\omega})$, such rotation laws have a special importance in the theory of rotating stars. Since the force is conservative, the rotation laws are often referred to as conservative also. We shall see below that conservative rotation laws are not only mathematically convenient but also stable, at least against axisymmetric perturbations. 
The other important equation is the equation of energy balance. In a radiative zone and in the absence of motions, the familiar equation $d L / d r=4 \pi r^{2} \rho \epsilon$ takes the form

$$
\operatorname{div} \mathbf{F}=\rho \epsilon
$$

where $\mathbf{F}$ is the radiative flux and $\epsilon$ is the rate of nuclear energy generation. In $\mathrm{Be}$ stars, $\epsilon$ can be neglected in radiative zones, since all the nuclear energy generation takes place in the convective core. Then (8) generalises to

$$
\underset{\gamma-1}{P}(\mathrm{v} \cdot \boldsymbol{\nabla}) \ln \left(P / \rho^{\gamma}\right)=-\operatorname{div} \mathbf{F}
$$

in the presence of motions, where $\gamma$ is the ratio of specific heats. Physically, this equation says that energy balance is partly maintained by the advection of entropy by the velocity field. In general, large-scale motions are required to maintain energy balance in a rotating star, essentially because rotation introduces an extra function $\Omega(\mathbf{r})$ into the equations (Baker \& Kippenhahn 1959).

In the absence of internal motions other than rotation, the equations of stellar structure are similar in form to those for non-rotating models, the main complication being the solution of Poisson's equation for the non-sphericallysymmetric gravitational field. Models of slowly-rotating polytropes were constructed as early as 1933 by Chandrasekhar, but the treatment of the large distortions produced by rapid rotation needed to await the development of computers (e.g. James 1964). All such calculations require a rapid and accurate solution of Poisson's equation and early methods worked better for polytropes than for real stars; it was particularly troublesome to construct models of low-mass main-sequence stars, which have a strong central density concentration. One way around that difficulty is to construct approximate, pseudo-spherical (one-dimensional) models. The most useful such technique was devised by Kippenhahn \& Thomas (1970) and also used by Papaloizou \& Whelan (1973). In the latter formulation the force balance equations are written:

$$
f_{1} \frac{d P}{d \eta}=-\rho \frac{G M}{\eta^{2}}+f_{2}, \quad \frac{d M}{d \eta}=4 \pi \eta^{2} \rho f_{3} .
$$

Here $\eta$ is a length, constant on a surface of constant total potential $\Psi$ (e.g. $\eta$ might be the polar radius of the $\Psi$-surface). The $f_{i}$ are correction factors, depending only on the geometry of the potential surfaces. The approximation consists of evaluating these three correction factors using the Roche approximation for the gravitational potential, so that:

$$
\Psi=\frac{G M}{r}+W
$$


This avoids solving the two-dimensional form of Poisson's equation. Although this method gives satisfactory results for uniform rotation, it becomes increasingly inaccurate as the degree of differential rotation is increased.

For studies of the effects of extreme differential rotation, there is no alternative to a full two-dimensional numerical calculation. The method of Kippenhahn \& Thomas (1970) and Papaloizou \& Whelan (1973) can be extended to two dimensions by iterating to improve the approximation for $\Psi$. If the Roche approximation is used as a first step, the resulting density distribution can be used to calculate a new approximation to the gravitational potential which can then be used to evaluate the correction factors in the next iteration. This is essentially the basis of the self-consistent field (SCF) method devised earlier by Ostriker \& Mark (1968), in which the equilibrium equations and Poisson's equation are solved alternately. Unfortunately this iterative scheme fails to converge even for a non-rotating model if the mass is less than about $9 M_{\odot}$ (Clement 1978). The SCF calculations were therefore restricted to stars earlier than about B2 on the main sequence. More recent calculations solve Poisson's equation simultaneously with the equilibrium equations (e.g. Clement 1978, 1979; Eriguchi \& Müller 1985a, b; Müller \& Eriguchi 1985) and these techniques seem to be able to cope with almost any density distribution, although expensive in computer time even using Fast Fourier Transforms to solve Poisson's equation (Clement 1986, Appendix). This makes it difficult to use full two-dimensional calculations to study stellar evolution, although Zorec (private communication; see also Zorec et al., this volume) is developing a two-dimensional code which should enable evolutionary calculations to be made.

We now turn to some of the results of numerical calculations.

\section{EFFECTS OF ROTATION ON OVERALL STRUCTURE}

The most obvious effect of rotation on a star is that the star is

flattened; for uniform (solid-body) rotation, its equatorial radius increases and its polar radius decreases. For uniform rotation, the calculations of Papaloizou \& Whelan (1973) using the Roche approximation gave polar radius changes of less than $3 \%$ but equatorial radius changes of up to $50 \%$, the maximum occurring when $\alpha=1$. It is this distortion of shape that is of most importance for the observable consequences of rotation (Collins \& Smith 1985) but its direct effects on the internal structure are small because of the small fraction of the stellar mass that is involved in the surface distortion. Unfortunately, even the effects of uniform rotation are somewhat uncertain; no exact two-dimensional calculations of uniformly-rotating stars appear to have been published, but Clement's work (1979) on the behaviour of stellar properties with the ratio of total rotational energy to gravitational energy for various degrees of differential rotation suggests 
that the changes in polar radius may be larger and those in equatorial radius smaller than given by the approximate calculations, even though the Roche approximation is adequate for determining the central conditions. This needs to be checked by new calculations.

The central conditions in a star are determined by the weight of the overlying layers. In a rotating star, the main effect of the centrifugal force is to reduce the effective gravity and the central conditions mimic those in a non-rotating star of lower mass. That is, the density is higher and the temperature and luminosity are lower than if the star were not rotating. This was realised in 1923 by Milne and has been quantitatively confirmed for uniformly rotating stellar models by many authors (e.g. Sackmann 1970, Papaloizou \& Whelan 1973). For the upper-main-sequence stars with which we are concerned here, the maximum effects for uniform rotation are quite small, giving a decrease in luminosity of $5-10 \%$ at the maximum rotation speed.

For differentially-rotating models, the situation is more complicated, the central pressure and temperature lying off the $P-T$ relation for non-rotating stars by an amount that depends in detail on the rotation speed and on the degree of differential rotation. Fortunately, there is a simplifying feature; at least for high-mass models ( $\geq 15 M_{\odot}$ ) (Bodenheimer 1971, Fig. 5) and probably also for low-mass models (Tuominen 1972, Fig. 5; Clement 1979, Fig. 1), the changes in central temperature and density and in luminosity for a star of a given mass depend only on the total angular momentum $J$ of the star and not on its distribution. For stars of different mass $M$, the ratio of the luminosity to that of a non-rotating star of the same mass varies approximately as $J / M^{2}$ (Tuominen 1972 , Fig. 6). If the angular velocity increases inwards, much more angular momentum can be stored in a differentially-rotating model than in a uniformly-rotating model with the same value of $\alpha$ and so rotation can have much larger effects - the luminosity may decrease by a factor of four or more (e.g. Clement 1979). For both uniform and non-uniform rotation, the fractional change in luminosity increases roughly as the square of the angular velocity. In the case of differential rotation, the limit is set not by $\alpha=1$ but by stability considerations; the rotational energy must be less than about $10 \%$ of the gravitational energy if the star is to be secularly stable (Clement 1979). All these results are for conservative rotation laws but are probably qualitatively the same for non-conservative laws.

Although we shall not be much concerned here with the shapes of rotating stars, it is worth noting that the shapes of differentially-rotating stars may be very different from those of uniformly-rotating stars, at least for the case of angular velocity increasing inwards. In the limit in which centrifugal force balanced gravity throughout the star (cf. Frank 1979), the star would resemble a toroid. Such structures may be relevant as models of thick accretion discs, although they are probably unstable (Papaloizou \& Pringle 1984, 1985). Realistic models with a 
significant internal pressure gradient may still show a deep 'dimple' in the polar regions and the equatorial radius may even be less than for the non-rotating star of the same mass. The polar radius changes can be up to $50 \%$ and the ratio $R_{\mathrm{e}} / R_{\mathrm{p}}$ may be as much as 3 or 4 . The contrast between such models and the familiar picture of a uniformly-rotating star can be seen by comparing Fig. 4 of Bodenheimer (1971) with Fig. 1 of Sackmann \& Anand (1970). Even more extreme pictures can be found in the papers by Eriguchi \& Müller (1985a, b), but such high degrees of differential rotation are unlikely to be present in real stars.

\section{THE ANGULAR MOMENTUM DISTRIBUTION}

Since the effects of rotation are much larger if the angular velocity increases inward than if it is uniform, an important question is: what do we expect the internal angular momentum distribution to be in stars? Unfortunately, as we shall see, there is no very satisfactory answer to that question, either from theory or from observation.

I shall consider first the theoretical constraints, restricting myself mainly to radiative regions without magnetic fields.

\section{Meridional circulation}

The first theoretical difficulty is that any arbitrarily imposed angular momentum distribution (AMD for short) will inevitably change with time as a result of large-scale motions which redistribute angular momentum. These motions arise in a region where radiation is the dominant mode of energy transfer because rotation distorts the temperature surfaces away from spheres and radiative equilibrium (equation (8)) no longer holds: for a given fluid element, the heat loss by radiation no longer balances the inflow of heat by radiation and the element either heats up or cools down with respect to its surroundings. Buoyancy forces then lead to a slow, large-scale axisymmetric circulation in meridional planes which restores thermal equilibrium (equation (9)). Molecular and radiative viscosity are negligible for stellar material (the typical viscous timescale is orders of magnitude longer than the Hubble time) and so fluid elements conserve angular momentum (equation (3)). The circulation therefore gradually alters the AMD, on a circulation timescale $\tau_{\text {circ }}$ of order $\tau_{\mathrm{KH}} / \alpha$, where $\tau_{\mathrm{KH}}\left(=G M^{2} / R L\right)$ is the Kelvin-Helmholtz or thermal timescale.

The breakdown of radiative equilibrium was first pointed out by von Zeipel (1924) for uniformly rotating stars and the consequent meridional circulation was discussed by Vogt (1925) and Eddington (1925, 1929). The first correct estimate of the circulation velocities was given by Sweet (1950). Other early work was reviewed by Mestel (1965) and there is a good physical discussion in 
Schwarzschild's book (1958). It was recognised by Baker \& Kippenhahn (1959) that circulation currents would arise quite generally for nearly all rotation laws, essentially because the imposition of a particular AMD puts an extra mathematical condition on the equations of stellar structure which is not in general compatible with the condition of radiative equilibrium. Roxburgh (1966, appendix) showed explicitly that there must be circulation for all conservative AMDs (those for which angular momentum is a function only of distance from the rotation axis).

\section{Possible steady states}

If circulation is constantly changing the AMD, is any steady state possible? In the absence of constraints, only two types of steady state can be imagined. In the first of these, we impose radiative equilibrium, in which case there is no circulation, and ask what (necessarily non-uniform and non-conservative) rotation law is required by that condition. This begs the question of whether an arbitrary initial AMD will evolve to a circulation-free state, but for a given mass of star there is a well-defined family of solutions, depending only on what boundary condition is adopted for $\Omega$ (Clement 1969). Marks \& Clement (1971) have argued for a particular boundary condition (but see Smith 1971b) and the corresponding unique solution was calculated by Harris \& Clement (1971). The angular velocity increases inwards by a modest factor, but the constant angular velocity surfaces are not simple and, as we shall see, the AMD is unstable.

The other conceivable steady state is one in which the angular momentum is constant on streamlines of the circulation, so the circulation does not change the AMD. No such models have been constructed, but the following simple argument (Randers 1941) shows that the only solutions are either trivial or unstable. Since the circulation streamlines must be closed, the pattern of streamlines of an axisymmetric circulation must be a nested set of loops in any meridional plane. The angular momentum per unit mass is $\Omega \tilde{\omega}^{2}$ and must in particular be constant on the largest loop, which must, by symmetry, run up the rotation axis, $\tilde{\omega}=0$. Then either $\Omega \tilde{\omega}^{2}=$ constant everywhere $=0$ and so $\Omega=0$ off the axis (the trivial solution), or $\Omega \tilde{\omega}^{2}$ varies from one streamline to the next. Because the streamlines are nested about some point, $h=\Omega \tilde{\omega}^{2}$ must then have a maximum or minimum somewhere in the interior and so there must be regions where $\partial h / \partial \tilde{\omega}<0$. This violates Rayleigh's criterion (1920) for the stability of a rotating incompressible liquid and we shall see that it also implies instability for the compressible fluid of a star. 


\section{Stability considerations}

The question of the stability of stellar AMDs is a complicated one, which is not completely settled. It is clear that there are certain rotation laws that are unstable, but the growth rates and the non-linear development of the instabilities remain a matter of debate. All I can do here is to try to summarise what is known and to indicate what are the unsettled questions.

Axisymmetric perturbations are the simplest and for that case there are firm results (cf. Moss \& Smith 1981). The first results were obtained for adiabatic perturbations by Solberg (1936) and Høiland (1939) and are discussed fully in the comprehensive article by Wasiutynski (1946). A stellar rotation law is stable against adiabatic, axisymmetric disturbances if and only if

$$
\frac{1 \partial h^{2}}{\tilde{\omega}^{3}} \frac{\partial \tilde{\omega}}{\partial \tilde{\omega}^{2}}-\nabla s>0
$$

and

$$
-\mathbf{g}_{\mathrm{eff}} \cdot \hat{\mathbf{z}}\left(\frac{\partial h^{2}}{\partial \tilde{\omega}} \frac{\partial s}{\partial z}-\frac{\partial h^{2}}{\partial z} \frac{\partial s}{\partial \tilde{\omega}}\right)>0
$$

where $h=\Omega \tilde{\omega}^{2}$ is the specific angular momentum, $\tilde{\omega}$ is the distance from the rotation axis, $g_{\text {eff }}=\mathbf{g}+\Omega^{2} \tilde{\boldsymbol{\omega}}$ is the sum of gravity and centrifugal force, $s=(1 / \gamma) \ln \left(P / \rho^{\gamma}\right)$ is related to the specific entropy and $\hat{\mathbf{z}}$ is a unit vector parallel to the rotation axis. These conditions are a generalisation to a rotating star of the familiar Schwarzschild criterion for convection, which can be written

$$
\mathrm{g}_{\mathrm{eff}} \cdot \nabla s>0 .
$$

That the conditions are both necessary and sufficient has been shown by Eliassen \& Kleinschmidt (1957) and independently by Fricke \& Smith (1971). Wasiutynski gave a simple geometrical interpretation, which shows that there is an unstable range of directions (towards the rotation axis, between $g_{e f f}$ and $\nabla s$ ) for the angular momentum vector $\nabla h^{2}$ (cf. Moss \& Smith 1981, Fig. 10). A simple extension of Randers' (1941) argument quoted above shows that for a steady state with angular momentum constant on closed streamlines, there must be some regions where $\nabla h^{2}$ points into the unstable range of directions. That steady state is therefore unstable on a dynamical timescale.

The circulation-free steady states can also be shown to be unstable if we consider more general perturbations. If the perturbations are adiabatic, buoyancy forces tend to stabilise motions in a radiative region. However, if we make a very slow perturbation, on a heat-leakage timescale, a displaced element has time to reach thermal equilibrium with its surroundings and so is no longer buoyant. A compressible fluid therefore behaves on a thermal timescale in a similar way to a 
liquid. A local stability analysis by Goldreich \& Schubert (1967) and by Fricke (1967, 1968) (see also Smith \& Fricke 1975) showed that the conditions

$$
\partial h^{2} / \partial \tilde{\omega} \geq 0, \quad \partial h^{2} / \partial z=0
$$

are necessary for stability against axisymmetric perturbations (it has not proved possible so far to decide whether they are sufficient). The first of these is identical to Rayleigh's criterion (1920) (when it is violated, exchanging neighbouring rings of material lowers the total energy if the rings conserve angular momentum) and the second is equivalent to the Taylor-Proudman theorem for an incompressible fluid (Greenspan 1968). Geometrically, the conditions are much more restrictive than the Solberg-Høiland conditions: angular momentum must be constant on cylinders parallel to the rotation axis and must not increase inward. That is sufficient to rule out all the circulation-free models.

However, the above discussion has only skimmed the surface of the very complex range of possible instabilities. A full discussion of the stability of rotation laws requires consideration of at least the following:

Thermal stratification (buoyancy forces)

$$
\text { (frequency: } \begin{aligned}
N_{\mathrm{T}}^{2} & =-\left(\nabla_{\mathrm{rad}}-\nabla_{\mathrm{ad}}\right) g / H_{\mathrm{p}} \\
& >0 \text { in radiatively stable regions) }
\end{aligned}
$$

Angular momentum gradient

$$
\text { (frequency: } \left.N_{\Omega}^{2}=\left(1 / \tilde{\omega}^{3}\right)\left(\partial h^{2} / \partial \tilde{\omega}\right)\right)
$$

Molecular weight gradient

$$
\begin{aligned}
& \text { (frequency: } N_{\mu}^{2}=-g(\partial \ln \mu / \partial r \text { ) } \\
& >0 \text { as a result of nuclear evolution. } \\
& N_{\mu}^{2} \text { is very small except near the edge of the convective core.) }
\end{aligned}
$$

Magnetic fields

Diffusion of:

$$
\text { (frequency: } N_{\mathrm{B}}^{2}=B^{2} / \mu_{o} \rho r^{2} \ll N_{\mathrm{T}}^{2} \text { in general) }
$$

$$
\begin{aligned}
& \text { heat (diffusivity } \kappa_{\mathrm{T}} \text { ) } \\
& \text { angular momentum (diffusivity } \nu=\text { viscosity) } \\
& \text { molecular weight (diffusivity } \kappa_{\mu} \text { ) } \\
& \text { magnetic field (diffusivity } \eta=\text { resistivity) } \\
& \text { In general, } \kappa_{\mathrm{T}}>>>>\nu \simeq \kappa_{\mu} .
\end{aligned}
$$

Shear flows

(both parallel and perpendicular to gravity)

Axisymmetric and non-axisymmetric perturbations

Baroclinic effects

(these arise when surfaces of constant $T$ and constant $P$ are not coincident and so either $\Omega \neq \Omega(\dot{\omega})$ or there is a $\mu$-gradient or both).

Combining these considerations clearly gives rise to an enormous range of 
possibilities which I cannot hope to summarise here and which are in any case not completely understood. Detailed discussion can be found in many papers in the astrophysical and fluid dynamical literature, of which the following is a small selection that I have found particularly useful: Acheson (1978), Knobloch (1982), Knobloch \& Spruit (1982, 1983), Spruit et al. (1983), Spruit \& Knobloch (1984), Zahn $(1983,1984)$. References to earlier work can be found in these papers and/or in Moss \& Smith (1981).

Leaving aside magnetic fields (which are carefully discussed by Acheson 1978), I shall attempt to give a brief overview of what $I$ see as the main recent results that are relevant to Be stars, putting the Solberg-Høiland and Goldreich-SchubertFricke (GSF) instabilities already discussed into a more general context.

\section{Stability in the absence of diffusion}

Neglecting all forms of diffusion, there are three instabilities to be considered, all of which grow on a dynamical timescale.

The Solberg-Høiland instability will occur if

$$
N_{\Omega}^{2}+N_{\mathrm{T}}^{2}<0
$$

and/or the geometrical condition illustrated in Fig. 10 of Moss \& Smith (1981) is satisfied. The $N_{\mathrm{T}}^{2}$ term represents the stabilising effect of buoyancy. Since there is no buoyancy stabilisation along constant pressure surfaces, a necessary condition for dynamical stability is

$$
\left(\frac{\partial h^{2}}{\partial \tilde{\omega}}\right)_{P=\text { const. }}>0
$$

Buoyancy also acts to stabilise shear flow instabilities and a vertical shear (which I shall represent for simplicity as a radial gradient) is stable so long as the Richardson criterion

$$
\left(r \frac{\partial \Omega}{\partial r}\right)^{2}<4 N_{\mathrm{T}}^{2}
$$

is satisfied. Horizontal shear is unaffected by gravity and so a sufficient condition for the stability of horizontal shear flow is that

$$
\frac{\partial^{2} h}{\partial \mu^{2}} \neq 0
$$


where $\mu$ is the cosine of the co-latitude. This is a generalisation of the condition for plane shear flow that the shear profile should contain no inflection points.

If pressure and entropy surfaces do not coincide (which means either a non-conservative rotation law or a molecular weight gradient, or both), non-axisymmetric motions in a direction between these surfaces release energy if they are of sufficiently long wavelength. Roughly speaking, that will be true only if $\Omega \simeq N_{\mathrm{T}}$, which occurs for $\Omega \simeq \Omega_{\text {crit }}$, i.e. for very rapid rotation, or for $N_{\mathrm{T}} \ll 1$ : near convection zone boundaries, where $\nabla=\nabla_{\mathrm{ad}}$. Because many of the usual approximations break down for rapid rotation and near convection zones, it is difficult to give a more precise discussion of this baroclinic instability.

A recent paper by Fujimoto (1986) has used a global analysis for a Boussinesq fluid to find sufficient conditions for stability that are a combination of a generalised Richardson condition and a geometrical condition that relates both to the variation of $\Omega$ along equal pressure and equal density surfaces and to the degree of baroclinicity. The two conditions can be satisfied simultaneously only for uniform rotation, and so uniform rotation is sufficient (but not necessary) for stability against adiabatic, non-axisymmetric perturbations, i.e. against an instability growing on a dynamical timescale. This suggests, but does not prove, that departures from uniform rotation in real stars are small.

\section{Effects of diffusion}

If a star is stable against instabilities that grow on a dynamical timescale, then we must ask whether there are other instabilities with slower growth rates. The relevant timescales are now those of the various possible diffusion processes. I shall discuss briefly three classes of instability that arise as a result of diffusion.

As discussed earlier, thermal diffusion removes the stabilising influence of buoyancy and so the Solberg-Høiland conditions (12) are replaced by the more restrictive GSF conditions (14) which require conservative rotation with angular momentum increasing outwards. However, viscous diffusion relaxes these conditions again, giving instability if

$$
N_{\Omega}^{2}=\frac{1}{\bar{\omega}^{3}} \frac{\partial h^{2}}{\partial \tilde{\omega}}<-\frac{\nu}{\kappa_{\mathrm{T}}} N_{\mathrm{T}}^{2}
$$

or

$$
\left(\frac{\partial \ln h^{2}}{\partial z}\right)^{2}>\frac{\nu}{\kappa_{\mathrm{T}}} \frac{g_{\mathrm{eff}}}{\Omega^{2} \bar{\omega}} \simeq \frac{\nu}{\kappa_{\mathrm{T}}} \frac{1}{\alpha} .
$$

This is an example of what fluid dynamicists call a double diffusive instability (Turner 1973). Because of an analogy in incompressible fluids containing salt and 
temperature gradients, in which the instability is observed to grow in the form of long 'fingers' of salty fluid, the GSF instability is sometimes referred to as the 'salt-finger' instability and the instability may initially produce long streamers of constant angular momentum before they are broken up by shearing. Since $\frac{\nu}{\kappa_{\mathrm{T}}} \simeq 10^{-6}$ for stars, the stabilising effect of viscosity is small except for slow rotation ( $\alpha \simeq 10^{-6}$, e.g. the Sun). For Be stars the standard GSF conditions are hardly changed.

If there is a molecular weight gradient, it can have a much more important stabilising effect, essentially by producing an effective buoyancy force that diffuses away only on the slow viscous timescale. The first equation in (19) gains a term and there is instability if

$$
N_{\Omega}^{2}<-\frac{\nu}{\kappa_{\mathrm{T}}} N_{\mathrm{T}}^{2}-\frac{\nu}{\kappa_{\mu}} N_{\mu}^{2}
$$

Since $\kappa_{\mu} \simeq \nu$, a small $\mu$-gradient is sufficient to stabilise and may permit a significant vertical shear near the boundary of the convective core in Be stars.

In the presence of diffusion, two completely new instabilities can arise. The first is a triply diffusive instability, which results from an interplay between $\kappa_{\mathrm{T}}, \nu$ and $\kappa_{\mu}$. In general, such an instability occurs if the destabilising agent (in this case an unstable angular momentum gradient) has a diffusivity $(\nu)$ intermediate between those of the two stabilising agents (here, temperature and molecular weight gradients). (In a related magnetic instability (Appendix to Acheson 1978), the destabilising agent is magnetic buoyancy while the stabilising agents are the angular momentum and temperature gradients.) Like the GSF instability, it is suppressed by quite a small molecular weight gradient. The other new instability is a double diffusive instability whose analogue is the thermohaline convection that occurs if a layer of water in which the salt concentration increases downwards is heated from below (Turner 1973). It sets in as an oscillation of growing amplitude at frequency $N_{\mathrm{T}}$. It occurs in baroclinic configurations (which in the presence of molecular weight gradients may not require non-conservative rotation), where motions in directions between the pressure and temperature surfaces find a stabilising angular momentum gradient but a destabilising temperature gradient. The unstable motions are axisymmetric and so the instability is known as the axisymmetric baroclinic diffusive (ABCD) instability. Because it requires a large molecular weight gradient to suppress it $\left(N_{\mu} \simeq N_{\mathrm{T}}\right)$, Spruit et al. (1983) have suggested that the $A B C D$ instability is important for mixing in the radiative interior of the Sun and would lead to a significant angular velocity gradient $\left(\Omega_{\mathrm{c}} / \Omega_{\mathrm{s}} \simeq 20\right)$; helioseismological data do not support such a large gradient (Duvall et al. 1984), although the results are highly uncertain for $r<0.3 R_{\odot}$.

In rapidly rotating stars $\left(\Omega / \Omega_{\text {crit }}>10^{-2}\right)$ these diffusive instabilities are less 
important, because the dominant role is played by shear flow and baroclinic instabilities. The effect of diffusion on the shear flow instabilities is rather uncertain, because turbulence is involved.

For horizontal shear, the typical horizontal scale is of the order of the stellar radius and so the appropriate Reynolds number is

$$
R_{\mathrm{e}} \simeq \frac{\Omega R_{*}^{2}}{\nu} \gg R_{\mathrm{e}}^{\mathrm{crit}}\left(\simeq 10^{3}\right)
$$

the small viscosity guarantees strong turbulence. However, the small viscosity also means that the growth time for the turbulence, $\tau_{\text {growth }} \simeq R_{*}^{2} / \nu$, is much greater than the stellar lifetime and so an infinitesimal perturbation will not grow fast enough for turbulent mixing of angular momentum along a pressure surface to be important. Sufficient conditions for stability are then equations (16) and (18), which are fairly unrestrictive. However, there are so many possible disturbances, arising for example from other instabilities, that it is quite likely that turbulence is either induced by finite amplitude perturbations or left over as 'fossil turbulence', having been generated by some other instability (e.g. a retreating convection zone or a vertical shear instability). Once any turbulence is present, it acts to produce an effective 'turbulent viscosity' many times larger than the molecular viscosity. This dramatically speeds up the growth rate of the viscous instability and causes a rapid smoothing out of the horizontal shear, leading to an angular velocity which is almost constant along a pressure surface, as first suggested by Zahn $(1974,1975)$. Once generated, the turbulence will decay only very slowly as a result of molecular viscous and thermal diffusion and so turbulent motions are likely to persist, with particularly large amplitude in the low-density surface layers. However, this discussion is certainly not rigorous, and Kippenhahn $\&$ Thomas (1981) have argued that the horizontal $\Omega$ distribution depends on the history of the star and need not be uniform.

The stability condition for vertical shear is also essentially unaffected by diffusion if only infinitesimal perturbations are considered. Again, however, finite amplitude perturbations in the form of turbulence give a quite different result, essentially by accelerating the destruction by thermal diffusion of buoyancy stabilisation. Following Zahn (1974), instability will occur if

$$
\left(r \frac{\partial \Omega}{\partial r}\right)^{2}>\frac{\nu}{\kappa_{\mathrm{T}}} R_{\mathrm{e}}^{\mathrm{crit}} N_{\mathrm{T}}^{2} \simeq 10^{-3} N_{\mathrm{T}}^{2}
$$

(cf. $4 N_{\mathrm{T}}^{2}$ in the absence of diffusion). However, a $\mu$-gradient can be stabilising and allow a larger vertical shear (cf. Spruit et al. 1983, equation (2)).

Timescales for the redistribution of angular momentum by these turbulent shear instabilities are $\tau_{\text {ver }} \simeq R_{\mathrm{e}}^{\mathrm{crit}} /|r d \Omega / d r|($ Zahn 1975) and (if the effective Reynolds 
number is equal to the critical value) $\tau_{\text {hor }} \simeq R_{e}^{\text {crit }} / \Omega$. Both are much shorter than the circulation timescale, $\tau_{\mathrm{KH}} / \alpha$, and so probably determine the rotation law; they may well also suppress the circulation.

\section{Deductions}

To try to draw any conclusions from this superficial discussion of a very complex problem is rather hazardous. However, I shall risk a few sweeping remarks, suitably hedged about with cautions.

If shear instabilities are dominant (and it is a large if), and if there is not a significant molecular weight gradient, then a possible rotation law seems to be

$$
\Omega \simeq \text { constant on pressure surfaces }
$$

and

$$
r \frac{d \Omega}{d r}<10^{-3 / 2} N_{\mathrm{T}} \simeq \frac{\Omega_{\text {crit }}}{30} .
$$

This corresponds to only a $3 \%$ departure from uniform rotation if $\Omega_{\text {surf }} \simeq \Omega_{\text {crit }}$ (a larger departure for slower rotation), which may be consistent with Fujimoto's (1986) result. Since magnetic fields also tend to maintain uniform rotation (essentially because they resist stretching and twisting), the best conjecture for main-sequence stars may be that

$$
\Omega \simeq \text { uniform }
$$

This would mean that Be star precursors were uniformly-rotating main sequence stars. Evolution may change this, so that observed Be stars do not have $\Omega$ uniform, but I would expect $\Omega$ still to be constant on pressure surfaces, since the redistribution timescale is very short.

Now come the cautions. First, $\Omega$ constant on pressure surfaces requires there to have been some initial turbulence or other similar finite-amplitude disturbance. Otherwise, constant angular momentum on pressure surfaces may be a better guess (cf. Kippenhahn \& Thomas 1981), or other instabilities may dominate, giving a more complicated and uncertain picture. However, if Fujimoto's (1986) sufficient condition is anywhere near being also necessary, then uniform rotation is a good approximation regardless of how it is achieved.

Second, I have not discussed magnetic effects in any detail and my remarks about them tending to give uniform rotation are purcly heuristic. However, recent arguments by Mestel \& Weiss (1986) tend to confirm my conclusions, unless the magnetic field is extremely weak. 
Third, I have not discussed at all the rotation law in the convective core. This is usually assumed to rotate like a solid body, but there are arguments (Tayler 1973) for believing that constant angular momentum is a better approximation than constant angular velocity. If so, the core could be quite distorted and affect the surface shape.

Fourth, there are no accurate numerical models with $\Omega$ constant on pressure surfaces, and even for uniform rotation there are no published models of real stars with a proper solution of Poisson's equation.

Since the theoretical understanding of the likely rotation law within stars is so unsatisfactory, can anything be said from observations?

\section{OBSERVATIONAL CONSTRAINTS}

Although rotation has clearly observable effects on a number of stellar properties, the changes in these effects as a result of different rotation laws are second-order and not easy to detect. There are several possible approaches, none of which has yet been successful.

Because rotation affects both the luminosity and the shape of a star, the magnitude and colour of a rotating star depend on the rotation speed and the inclination of the rotation axis to the line of sight and so rotation produces a spread in the colour-magnitude diagram of a star cluster (Collins 1970, Moss \& Smith 1981). The amount of the spread for a given observed range of rotation speeds depends on the internal rotation law, since strong differential rotation corresponds to a larger total angular momentum than does uniform rotation at the same surface rotation speed. However, it is not possible uniquely to determine the rotation law from the observations, even in principle (Collins \& Smith 1985), and in practice the observational uncertainties are in any case too great for any very useful constraints to be applied (e.g. Smith 1971a). The best one can do is to rule out extreme differential rotation (Peacock \& Smith 1986): $\Omega_{\mathrm{p}}<4 \Omega_{\mathrm{e}}$.

Most measurements of rotation speeds come from Doppler half-widths, which are a fairly crude measure of the line profile. In principle, if the rotation speed varies over a stellar surface that variation should be reflected in the detailed shape of a spectral line and so careful analysis of line profiles should be able to reveal the surface differential rotation, though of course they can say nothing about the internal distribution of $\Omega$. Attempts to determine differential rotation from line profiles have been made by Stoeckley and his collaborators (Stoeckley 1968, Stoeckley et al. 1984, Stoeckley \& Buscombe 1986) and in the Fourier domain by Gray $(1977,1982)$. Their results are not conclusive, but Gray suggests that any departure from uniform angular velocity is not large, which is consistent with what the theoretical studies suggest. Stoeckley \& Buscombe (1986) claim 
differential rotation with $\Omega_{\mathrm{p}} / \Omega_{\mathrm{e}}$ up to about 1.6 but do not discuss the effect of turbulence on their line profiles.

A completely different approach is to ask what the differential rotation is on the surface of Jupiter, one of the very few rapidly rotating gaseous bodies ( $\Omega \simeq \Omega_{\text {crit }} / 3$ ) for which we can actually study the surface in detail. The belts, seen clearly also on Saturn, suggest a pattern dominated by shear flows, which may be some support for shear flow instabilities being the dominant factor in rotating stars. Perhaps more significantly, the pole-equator difference in angular velocity for Jupiter is only a few per cent, again consistent with what theory suggests for stars.

Ultimately, we should like to be able to 'observe' what is happening inside stars. Until very recently, this seemed an unrealisable goal. However, with the advent of helioseismology (Deubner \& Gough 1984, Gough 1986), it is now becoming possible to probe the Sun's internal structure by examining the frequencies of hundreds of acoustic (p-mode) oscillations of different spherical harmonic degree $l$ and resonant mode number $n$ (see Gough 1985 for a clear non-technical discussion). Although these modes are observed at the surface, and the modes of high $l / n$ are largely confined to the surface layers, it is known from theory that lower-degree modes penetrate throughout the Sun and their frequencies depend on the density distribution within the Sun (more directly, on the sound speed). It is therefore possible to invert the oscillation data to obtain the run of sound speed within the Sun and fairly good agreement has been obtained with the theoretical results from standard solar models (Christensen-Dalsgaard et al 1985). The necessary oscillation data can be obtained from spatially unresolved whole-disc Doppler data and so in principle can also be obtained for other stars. Such data are beginning to be obtained (Gough 1985, 1986, Guenther \& Demarque 1986). For the Sun, the further step has been taken of measuring the rotational frequency splitting of the oscillations. Detailed spatially-resolved observations (Duvall \& Harvey 1984) have been used to deduce the solar rotation as a function of depth (Duvall et al. 1984) and of latitude (Brown 1985). Except within $0.3 R_{\odot}$, where the uncertainties become large and it is not clear whether to believe the apparent sharp increase in $\Omega$ towards the centre (Libbrecht 1986), the results are consistent with uniform rotation, with the latitudinal variation in $\Omega$ smaller in the radiative interior than at the surface. It is not possible to make spatially-resolved measurements for other stars, but some information can in principle be obtained from whole-disc measurements (Dziembowski 1984) and this technique holds out great hope for the future of stellar rotation.

\section{CONCLUSIONS}

Despite a great deal of work on large-scale circulation and 
instabilities, there is still no clear theoretical expectation for the angular momentum distribution inside stars. However, the best guess at the moment seems to be uniform rotation, or very mild differential rotation with angular velocity constant on pressure surfaces.

There are too many uncertainties in interpreting observational data for there to be any direct observational constraints yet available, but all the existing data are consistent with uniform or nearly uniform rotation. Within the next ten years, asteroseismology may give us a direct determination of the run of angular velocity within a star other than the Sun.

If stars do rotate uniformly, the effect on the total luminosity is small, less than $10 \%$. However, the spread in observed luminosity and colour is larger, because of the shape distortion, and can have significant effects on colour-magnitude diagrams.

Although there are many models of rotating stars, very few are accurate in the sense that Poisson's equation is properly solved and there are no models of rapidly-rotating stars with non-conservative rotation laws. Surprisingly, there also seem to be no realistic models of uniformly-rotating stars for which Poisson's equation is fully solved.

Finally, if shear flow instabilities dominate the redistribution of angular momentum inside stars, and there is associated turbulence, the turbulent motions in the surface layers might act in a way analogous to the solar convection zone and be a source for at least some of the mechanical energy flux that seems to be needed to explain the hot winds observed in Be and related stars.

\section{Acknowledgement}

I am very grateful to Roger Discombe of the University of Sussex Computer Centre for his invaluable assistance in preparing the camera-ready text.

APPENDIX: $\alpha_{\max }$

If we make the Roche approximation for the gravitational potential,

then:

$$
\begin{aligned}
\Psi & =\frac{G M}{r}+\int_{0}^{\tilde{\omega}} \Omega^{2} \tilde{\omega} d \tilde{\omega} \\
& =G M\left[\frac{1}{r}+\frac{\Omega_{\mathrm{p}}^{2} R_{\mathrm{e}}^{2}}{G M} I(y)\right], \text { say, where } y=\tilde{\omega} / R_{\mathrm{e}}
\end{aligned}
$$

Then, since $I(y)=0$ at the pole, 


$$
\frac{1}{R_{\mathrm{p}}}=\frac{1}{R_{\mathrm{e}}}\left[1+\frac{\Omega_{\mathrm{p}}^{2} R_{\mathrm{e}}^{3}}{G M} I(1)\right]
$$

which we can re-write as

$$
x=1+\lambda x^{3}
$$

where $x=R_{\mathrm{e}} / R_{\mathrm{p}}, \lambda=\frac{\Omega_{\mathrm{p}}^{2} R_{\mathrm{p}}^{3}}{G M} I(1)$, and $I(1)$ depends on the internal rotation law The possible solutions of this cubic can be visualised by sketching the graphs of the functions $x$ and $1+\lambda x^{3}$ and looking for the intersections. In general there are three, of which the one of interest is the smaller positive solution. It is clear from a sketch (exercise for the reader!) that for large enough $\lambda$ there is no positive root and that the maximum value of $\lambda$ for which a root exists occurs when the straight line is a tangent to the curve, that is when:

$$
\begin{gathered}
\frac{d}{d x}\left(1+\lambda x^{3}\right)=1 \\
\text { i.e. } 3 \lambda x^{2}=1 \text { or } x^{2}=\frac{1}{3 \lambda} .
\end{gathered}
$$

Substituting this value of $x$ into the cubic gives:

$$
(1 / 3 \lambda)^{1 / 2}=1+\lambda(1 / 3 \lambda)^{3 / 2}
$$

which yields the maximum values of $\lambda$ and $x$ :

$$
\lambda_{\max }=4 / 27, \quad x_{\max }=3 / 2 .
$$

Hence we find

$$
\begin{aligned}
\alpha_{\max } & =\left(\frac{\Omega_{\mathrm{e}}^{2} R_{\mathrm{e}}^{3}}{G M}\right)_{\max }=\frac{\lambda_{\max }}{I(1)}\left(\frac{\Omega_{\mathrm{e}}}{\Omega_{\mathrm{p}}}\right)^{2} x_{\max }^{3} \\
& =\frac{1}{2} \frac{1}{I(1)}\left(\frac{\Omega_{\mathrm{e}}}{\Omega_{\mathrm{p}}}\right)^{2}\left\{\begin{array}{l}
=1 \text { for uniform rotation } \\
<1 \text { for } \Omega_{\mathrm{e}}<\Omega_{\mathrm{p}}
\end{array}\right.
\end{aligned}
$$

\section{REFERENCES}

Acheson, D.J. (1978). Phil. Trans. R. Soc., A289, 459-500.

Baker, N. \& Kippenhahn, R. (1959). Z. Astrophys., 48, 140-54.

Bodenheimer, P. (1971). Astrophys. J., 167, 153-63.

Brown, T.M. (1985). Nature, 317, 591-4. 
Chandrasekhar, S. (1933). Mon. Not. R. astr. Soc., 93, 390-405.

Chandrasekhar, S. (1969). Ellipsoidal Figures of Equilibrium. New Haven: Yale University Press.

Christensen-Dalsgaard, J., Duvall, T.L. Jr, Gough, D.O., Harvey, J.W. \& Rhodes, E.J. Jr (1985). Nature, 315, 378-82.

Clement, M.J. (1969). Astrophys. J., 156, 1051-68.

Clement, M.J. (1978). Astrophys. J., 222, 967-75.

Clement, M.J. (1979). Astrophys. J., 230, 230-42.

Clement, M.J. (1986). Astrophys. J., 301, 185-203.

Collins, G.W. II (1970). In Stellar Rotation, IAU Colloq. No. 4, ed. A. Slettebak, pp. 85-108. Dordrecht: D. Reidel.

Collins, G.W. II (1986). In Physics of Be Stars, IAU Colloq. No. 92, ed. A. Slettebak (this volume). Cambridge: Cambridge University Press.

Collins, G.W. II \& Smith, R.C. (1985). Mon. Not. R. astr. Soc., 213, 519-52.

Deubner, F.-L. \& Gough, D.O. (1984). Ann. Rev. Astron. Astrophys., 22, 593-619.

Durisen, R.H. \& Tohline, J.E. (1985). In Protostars and Planets II, eds D.C. Black \& M.H. Matthews, pp. 534-75. Tucson: University of Arizona Press.

Duvall, T.L. Jr, Dziembowski, W.A., Goode, P.R., Gough, D.O., Harvey, J.W. \& Leibacher, J.W. (1984). Nature, 310, 22-5.

Duvall, T.L. Jr \& Harvey, J.W. (1984). Nature, $\underline{310}$, 19-22.

Dziembowski, W. (1984). Adv. Space Res., 4, 143-50.

Eddington, A.S. (1925). Observatory, 48, 73-5.

Eddington, A.S. (1929). Mon. Not. R. astr. Soc., $\underline{90}, 54-8$.

Eliassen, A. \& Kleinschmidt, E. (1957). Handb. Phys., 48, 1-154.

Eriguchi, Y. \& Müller, E. (1985a). Astron. Astrophys., 146, 260-68.

Eriguchi, Y. \& Müller, E. (1985b). Astron. Astrophys., 147, 161-68.

Frank, J. (1979). Mon. Not. R. astr. Soc., 187, 883-904.

Fricke, K. (1967). Doctoral Dissertation, Goettingen.

Fricke, K. (1968). Z. Astrophys., 68, 317-44.

Fricke, K.J. \& Kippenhahn, R. (1972). Ann. Rev. Astron. Astrophys., 10, 45-72.

Fricke, K.J. \& Smith, R.C. (1971). Astron. Astrophys., 15, 329-31.

Fujimoto, M.Y. (1986). Astron. Astrophys., submitted (Preprint MPA 246,

Max-Planck-Institut für Physik und Astrophysik, Garching, July $1986)$.

Galileo Galilei (1612). Letters on Sunspots. English translation by Stillman Drake (1957) in Discoveries and Opinions of Galileo. New York: Doubleday Anchor Books.

Goldreich, P. \& Schubert, G. (1967). Astrophys. J., 150, 571-87.

Gough, D.O. (1985). Nature, News and Views, 314, 14-5. 
Gough, D.O. (1986). In Highlights of Astronomy, I, ed. J.-P. Swings, pp. 283-93. Dordrecht: D. Reidel.

Gray, D.F. (1977). Astrophys. J., 211, 198-206.

Gray, D.F. (1982). Astrophys. J., 258, 201-8.

Greenspan, H.P. (1968). The Theory of Rotating Fluids. Cambridge: Cambridge University Press.

Guenther, D.B. \& Demarque, P. (1986). Astrophys. J., 301, 207-12.

Harris, W.E. \& Clement, M.J. (1971). Astrophys. J., 167, 321-5.

Høiland, E. (1939). Archiv. Math. Naturvidensk., 46, Nr 5, 1-69.

James, R.A. (1964). Astrophys. J., 140, 552-82.

Kippenhahn, R. \& Thomas, H.-C. (1970). In Stellar Rotation, IAU Colloq. No. 4, ed A. Slettebak, pp. 20-9. Dordrecht: D. Reidel.

Kippenhahn, R. \& Thomas, H.-C. (1981). In Fundamental Problems in the

Theory of Stellar Evolution, IAU Symp. No. 93, eds D.Q. Lamb,

D.N. Schramm \& D. Sugimoto, pp. 237-56. Dordrecht: D. Reidel.

Knobloch, E. (1982). Geophys. Astrophys. Fluid Dynamics, 22, 133-58.

Knobloch, E. \& Spruit, H.C. (1982). Astron. Astrophys., 113, 261-8.

Knobloch, E. \& Spruit, H.C. (1983). Astron. Astrophys., 125, 59-68.

Lebovitz, N.R. (1967). Ann. Rev. Astron. Astrophys., 5, 465-80.

Lebovitz, N.R. (1975). In Problems of Stellar Hydrodynamics, Liège Colloq. 19,

Mem. Soc. R. Sciences Liège, $6^{e}$ Série, $8,47-53$.

Lebovitz, N.R. (1979). Ann. Rev. Fluid Mech., 11, 229-46.

Libbrecht, K.G. (1986). Nature, 319, 753-5.

Marks, D.W. \& Clement, M.J. (1971). Astrophys. J. Lett., 166, L27-9.

Mestel, L. (1965). In Stellar Structure, ed. L. Aller \& D.B. McLaughlin, pp.

465-97. Chicago: University of Chicago Press.

Mestel, L. \& Weiss, N.O. (1986). Mon. Not. R. astr. Soc., submitted.

Milne, E.A. (1923). Mon. Not. R. astr. Soc., 83, 118-47.

Moss, D. \& Smith, R.C. (1981). Rep. Prog. Phys., 44, 831-91.

Müller, E. \& Eriguchi, Y. (1985). Astron. Astrophys., 152, 325-35.

Ostriker, J.P. \& Mark, J.W.-K. (1968). Astrophys. J., 151, 1075-88.

Papaloizou, J. \& Pringle, J.E. (1984). Mon. Not. R. astr. Soc., 208, 721-50.

Papaloizou, J. \& Pringle, J.E. (1985). Mon. Not. R. astr. Soc., 213, 799-820.

Papaloizou, J.C.B. \& Whelan, J.A.J. (1973). Mon. Not. R. astr. Soc., 164, 1-10.

Peacock, T. \& Smith, R.C. (1986). Observatory, submitted.

Randers, G. (1941). Astrophys. J., 94, 109-23.

Rayleigh, Lord (1920). Scientific Papers, $\underline{6}$, 447-53.

Roxburgh, I.W. (1966). Mon. Not. R. astr. Soc., 132, 201-15.

Sackmann, I.-J. (1970). Astron. Astrophys., 8, 76-84.

Sackmann, I.-J. \& Anand, S.P.S. (1970). Astrophys. J., 162, 105-24.

Schlesinger, F. (1910). Publ. Allegheny Observatory, 1, 123-34. 
Schwarzschild, M. (1958). Structure and Evolution of the Stars. Princeton:

Princeton University Press.

Slettebak, A. (1976). In Be and Shell Stars, IAU Symp. No. 70, ed. A. Slettebak, pp. 123-36. Dordrecht: D. Reidel.

Smith, R.C. (1971a). Mon. Not. R. astr. Soc., 151, 463-83.

Smith, R.C. (1971b). Mon. Not. R. astr. Soc., 153, 33P-35P.

Smith, R.C. \& Fricke, K.J. (1975). Mon. Not. R. astr. Soc., 172, 577-84.

Solberg, H. (1936). Procès-Verbaux Ass. Météor., UGGI, Mem. et Disc., 2, 66-82.

Spruit, H.C., Knobloch, E. \& Roxburgh, I.W. (1983). Nature, 304, 520-2.

Spruit, H.C. \& Knobloch, E. (1984). Astron. Astrophys., 132, 89-96.

Stoeckley, T.R. (1968). Mon. Not. R. astr. Soc., 140, 121-39.

Stoeckley, T.R., Carroll, R.W. \& Miller, R.D. (1984). Mon. Not. R. astr. Soc, 208, 459 and Microfiche MN208/1.

Stoeckley, T.R. \& Buscombe, W. (1986). Mon. Not. R. astr. Soc., to appear.

Strittmatter, P.A. (1969). Ann. Rev. Astron. Astrophys., 7, 665-84.

Sweet, P.A. (1950). Mon. Not. R. astr. Soc., 110, 548-58.

Tassoul, J.-L. (1978). Theory of Rotating Stars. Princeton: Princeton University Press.

Tayler, R.J. (1973). Mon. Not. R. astr. Soc., 165, 39-52.

Tuominen, I.V. (1972). Ann. Acad. Sci. Fennicae, Sér. A, VI Physica, No. 392.

Turner, J.S. (1973). Buoyancy Effects in Fluids. Cambridge:

Cambridge University Press.

Vogt, H. (1925). Astron. Nachr., No. 5342, 223, 229-32.

von Zeipel, H. (1924). Mon. Not. R. astr. Soc., 84 , 665-83.

Wasiutynski, J. (1946). Astrophys. Norvegica, $\underline{4}, 1-497$.

Zahn, J.-P. (1974). In Stellar Instability and Evolution, IAU Symp. No. 59, eds P. Ledoux, A. Noels \& A.W. Rodgers, pp. 185-95. Dordrecht: D. Reidel.

Zahn, J.-P. (1975). In Problems of Stellar Hydrodynamics, Liège Colloq. No. 19, Mem. Soc. R. Sci. Liège, $6^{e}$ Sér., $\underline{8}, 31-4$.

Zahn, J.-P. (1983). In Astrophysical Processes in Upper Main Sequence Stars,

13th Saas-Fee Advanced Course, eds. A. Maeder \& B. Hauck, pp. 255-329. Sauverny: Geneva Observatory.

Zahn, J.-P. (1984). In Theoretical Problems in Stellar Stability and Oscillations,

Liège Colloq. No. 25, eds. A. Noels \& M. Gabriel, pp. 407-418.

Cointe-Ougrée: Université de Liège.

\section{Erratum}

The argument in the Appendix is incorrect (T.R. Stoeckley, private communication). Section 2 of the paper (The Maximum Value of $\alpha$ ) should therefore be ignored. 


\section{DISCUSSION FOLLOWING SMITH}

Zorec:

In our calculations of models of differentially rotating stars, we have used the Clement's method to solve the Poisson's equation. As in this method the mass of the star is a solution of the problem, we have used some general laws concerning the differentially rotating stars, to reduce the number of iterations needed to obtain a model for a given mass and a given deformation parameter (deformation parameter = rotational kinetic energy/gravitational potential energy). Using this procedure, we are preparing now the calculation of the evolution of stars in cylindrical differential rotation.

Smith:

It would be very useful to have evolutionary calculations with accurate models.

Marlborough:

I believe Tassoul has claimed that circulation velocities go to zero at the surface of a rotating star. Can you comment on the possible relevance of this?

Smith:

The circulation velocities can be made to go zero at the surface by a proper treatment of the viscous boundary layer, as shown by B.L. Smith, or by a suitable choice of the rotation law. If shear flow instabilities dominate, the circulation does not in any case play a significant role in determining the rotation law. However, Tassoul finds that a turbulent viscosity is necessary for a self-consistent model, so even zero circulation velocities may not imply zero observed velocities - turbulence may be seen at the surface.

\section{Underhill:}

You argue that there may be a large source of mechanical energy in the subphotospheric layers of a star and you speculate that this energy may be a source for the heat observed by means of "hot winds". There must be some method of coupling the energy from "below" to far "above" in the stellar mantle. Do you have any candidates for the method and any idea of its efficiency?

Smith:

I have no definite candidate, but would expect magnetic fields to be involved, perhaps via magneto-acoustic waves. Since we do not yet fully understand the mechanism of coronal heating in the sun, I think we can only argue that the mechanism for Be stars is likely to be related to that for the sun, and try to understand that first.

Collins:

If turbulence is established in the outer layers, wouldn't the speeds be so large as to be seen in line profiles?

Smith:

That is certainly possible. But the uncertainties in the theory of the turbulence generated by shear flow instabilities make it hard to estimate reliably the turbulent velocities. It may be better to use observed limits on turbulent velocities to constrain the shear flow models. The main expectation is for velocities which make the turbulent Reynolds number of the order of the critical Reynolds number. 